University of Nebraska - Lincoln

DigitalCommons@University of Nebraska - Lincoln

Biological Systems Engineering: Papers and

Publications

Biological Systems Engineering

2015

\title{
Identifying Intracellular pDNA Losses From a Model of Nonviral Gene Delivery
}

Timothy Michael Martin

University of Nebraska-Lincoln, timothy.michael.martin@gmail.com

Beata Joanna Wysocki

University of Nebraska-Lincoln

Tadeusz Antoni Wysocki

University of Nebraska-Lincoln

Angela K. Pannier

University of Nebraska-Lincoln, apannier2@unl.edu

Follow this and additional works at: https://digitalcommons.unl.edu/biosysengfacpub

Part of the Bioresource and Agricultural Engineering Commons, Environmental Engineering Commons, and the Other Civil and Environmental Engineering Commons

Martin, Timothy Michael; Wysocki, Beata Joanna; Wysocki, Tadeusz Antoni; and Pannier, Angela K., "Identifying Intracellular pDNA Losses From a Model of Nonviral Gene Delivery" (2015). Biological Systems Engineering: Papers and Publications. 423.

https://digitalcommons.unl.edu/biosysengfacpub/423

This Article is brought to you for free and open access by the Biological Systems Engineering at DigitalCommons@University of Nebraska - Lincoln. It has been accepted for inclusion in Biological Systems Engineering: Papers and Publications by an authorized administrator of DigitalCommons@University of Nebraska Lincoln. 


\title{
Identifying Intracellular pDNA Losses From a Model of Nonviral Gene Delivery
}

\author{
Timothy Michael Martin, Beata Joanna Wysocki, Tadeusz Antoni Wysocki, Senior Member, IEEE, and \\ Angela K. Pannier*
}

\begin{abstract}
Nonviral gene delivery systems are a type of nanocommunication system that transmit plasmid packets (i.e., pDNA packets) that are programmed at the nanoscale to biological systems at the microscopic cellular level. This engineered nanocommunication system suffers large pDNA losses during transmission of the genetically encoded information, preventing its use in biotechnological and medical applications. The pDNA losses largely remain uncharacterized, and the ramifications of reducing pDNA loss from newly designed gene delivery systems remain difficult to predict. Here, the pDNA losses during primary and secondary transmission chains were identified utilizing a MATLAB model employing queuing theory simulating delivery of pEGFPLuc transgene to HeLa cells carried by Lipofectamine 2000 nonviral DNA carrier. Minimizing pDNA loss during endosomal escape of the primary transmission process results in increased number of pDNA in the nucleus with increased transfection, but with increased probability of cell death. The number of pDNA copies in the nucleus and the amount of time the pDNAs are in the nucleus directly correlates to improved transfection efficiency. During secondary transmission, pDNAs are degraded during distribution to daughter cells. Reducing pDNA losses improves transfection, but a balance in quantity of nuclear pDNA, mitosis, and toxicity must be considered in order to achieve therapeutically relevant transfection levels.
\end{abstract}

Index Terms-Biological systems modeling, communication networks, molecular communication, nanobioscience, nonviral gene delivery, pDNA packet loss.

\section{INTRODUCTION}

$\mathbf{N}$ ANOSCALE communication networks [1]-[3] are usually defined as networks where at least one of the transmitter, receiver, medium or message carrier or one of their essential components have nanoscale $(1-100 \mathrm{~nm})$ dimensions [4]. Even though human cells are not that small, their

Manuscript received October 15, 2014; accepted January 12, 2015. Date of publication January 23, 2015; date of current version May 29, 2015. This work was supported in part by funds from the American Heart Association, the University of Nebraska Foundation (Layman Funds), the Nebraska Research Initiative, National Science Foundation, and USDA CSREES-Nebraska (NEB-21146). Asterisk indicates corresponding author.

T. M. Martin is with the Department of Biological Systems Engineering, University of Nebraska-Lincoln, Lincoln, NE 68583 USA (e-mail: timothy.michael. martin@gmail.com).

B. J. Wysocki and T. A. Wysocki are with the Department of Computer and Electronics Engineering, University of Nebraska-Lincoln, Omaha, NE 68182 USA (e-mail: beata.wysocki@unl.edu; twysocki2@unl.edu).

*A. K. Pannier is with the Department of Biological Systems Engineering, University of Nebraska-Lincoln, Lincoln, NE 68583 USA (e-mail: apannier2@unl.edu).

Color versions of one or more of the figures in this paper are available online at http://ieeexplore.ieee.org.

Digital Object Identifier 10.1109/TNB.2015.2392777 ligand receptors, endosomes, and molecular factors, which are essential components in communication processes, have nanoscale dimensions and these processes are critical in many cellular applications [3], [5]-[8], including gene delivery [6]. Gene delivery approaches serve as a platform to modify gene expression of a cell population with applications including functional genomics, tissue engineering, and gene therapy. While the use of modified viruses in the form of viral vectors efficiently deliver DNA, their numerous safety drawbacks such as toxicity, immunogenicity, mutagenesis, and tumorigenic effects make them less than desirable for many in vivo or ex vivo applications [9]-[11]. These problems with viral delivery have led to the development of nonviral gene delivery strategies, where genetic information encoded as plasmid DNA (pDNA) is transferred to cells with the help of a DNA carrier such as a lipid or polymer, together forming a pDNA packet.

A major problem preventing use of nonviral gene delivery systems in those applications is that large losses of the pDNAs occur after internalization into cells due to degradation in primary or secondary transmission chains. Primary transmission chains describe routing of the pDNA packet through a series of networked intracellular barriers from the plasma membrane to the cell nucleus: internalization into endosome, escape from endosome, translocation to nucleus, nuclear entry, and transcription/translation of the encoded transgenic protein [12]-[25]. During those steps, the pDNA can be degraded in the lysosome (if the pDNA packet fails to escape the endolysosome) or degraded in the cytoplasm after the pDNA escapes the endosome and unpacks during routing to the nucleus. Secondary transmission chains describe routing of the pDNA or pDNA packets during mitosis, where each cell splits into two daughter cells, and as a result, any pDNA or pDNA packets within the cell are also distributed to each of the two daughter cells. Degradation during secondary transmission has been never reported, although in a recent report, in cells where unpacked pDNA was microinjected into the cell nucleus, 28 percent of cells were unable to retain more than $75 \%$ of microinjected pDNA in the nuclei of daughter cells [26]. The result suggests either misdirecting and/or degradation of the pDNA during mitosis since the pDNA is again exposed to the potential degradation in the cytoplasm by nucleases. Reducing pDNA losses during primary and secondary transmission can be achieved through physiochemical modification of the gene delivery system [27]-[30]. However, the ramifications of design changes on improved transfection for new gene delivery systems remain difficult to predict. 
Efficient communication between macro- and nanocommunication systems and among nanosystems themselves can be achieved by using models to identify losses and to improve performance in the system, including nonviral gene delivery systems [7], [31], [32]. Several mathematical and computational models of nonviral gene delivery have been developed to provide an understanding of the intracellular distribution and kinetics of the pDNA [15], [22], [33]-[38]. However, quantitative relationships for pDNA packet loss and transfection remain unknown and prevent rationale design of delivery systems with improved performance [39]. We have recently developed a model of the pDNA packet transmission process in SIMULINK [40], using queuing theory [41]. In our telecommunications model, routing of the pDNA packets in primary and secondary transmission chains is considered the same as data packet transfer in a packet-switched network [42]. The model has been since improved by taking into account biological effects like mitosis and treatment-induced necrosis, which have been reported to affect transfection but have never been included in a model of nonviral gene delivery before [21], [24], [43], [44]. That improved model was implemented in MATLAB and simulation results showed significant agreement with experimental data, including pDNA nuclear internalization and transfection efficiency [42], and thus validated the model. In this work, we have further refined our telecommunications model [42] by applying equivalent $M / M / 1$ queues instead of $M / M / \infty$ queues and then used the model to identify and characterize, for the first time, losses incurred by the degradation of the pDNA in primary and secondary transmission. Additionally, this paper provides mathematical derivation of the probabilities of service for queues used in the model and heuristics derived from the experimental data, including probability of necrosis due to toxicity and probability of transfection as a function of the number of nuclear plasmids, which could serve as important considerations for design of future carriers.

The remainder of this paper is organized as follows: In Section II we provide theoretical aspects of our model. In Section III we describe the baseline performance of the model of nonviral delivery considered as a telecommunications network. In Section IV, we evaluate the effect of the quantity of pDNAs in the nucleus and the length of time of the pDNAs in the nucleus on the primary transmission losses in the system. Additionally, we characterize pDNA packet loss at the endosomal escape stage due to lysosomal degradation in terms of transfection. In Section $\mathrm{V}$, we evaluate secondary transmission pDNA losses that occur during mitosis. Finally, in Section VI we draw conclusions based on this work.

\section{Theoretical AsPects of Model}

Queuing theory has previously been used to describe data communication networks [45], servicing of patients at hospitals [41], and the HIV infection process [46]. In our model, nonviral gene delivery was modeled as a digital process because events, such as the number of internalized pDNA packets or pDNAs, occur as integer numbers. The signals considered in nonviral gene delivery come from a very small alphabet of symbols, i.e., pDNAs, pDNA packets, transgenic mRNAs, and transgenic

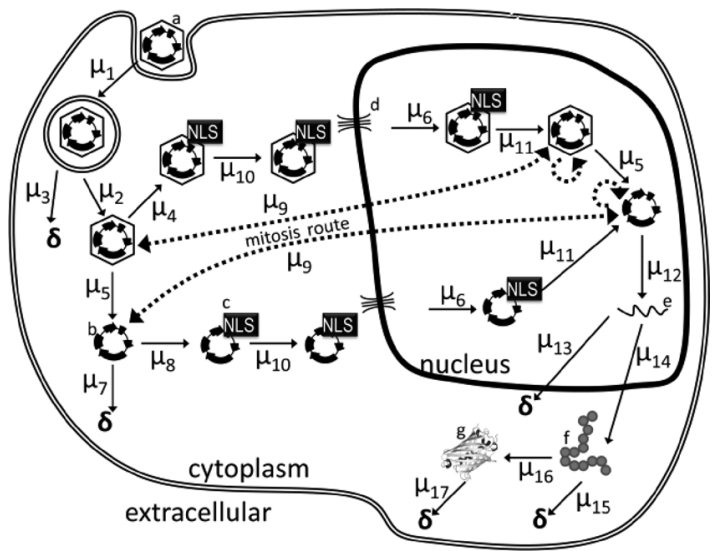

Fig. 1. Routing of pDNA packets after arrival to cells. The pDNA will route differently in the packed state (i.e., as a pDNA packet), unpacked state (i.e., pDNA alone), or if the cell is undergoing mitosis. Routes that are active during mitosis are indicated by dashed lines. Kinetic parameters are reported in [42]. ${ }^{a}$ pDNA packets; ${ }^{b}$ unpacked pDNA; ${ }^{\mathrm{c}}$ nuclear import binding proteins; ${ }^{\mathrm{d}}$ nuclear pore complex; ${ }^{\mathrm{e}} \mathrm{mRNA}$; ${ }^{\mathrm{f}}$ unfolded protein; ${ }^{\mathrm{g}}$ folded protein; $\delta$ indicates degradation. Reprinted with permission from John Wiley and Sons under license number 3415431409731 [42].
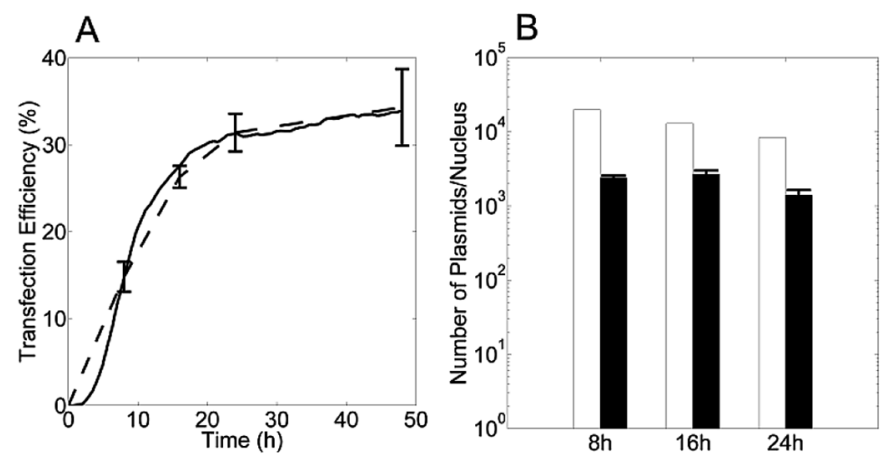

Fig. 2. Model output (solid line in (A); or white bars in (B) was not statistically different from in vitro results (dashed line in (A); or black bars in (B) at the indicated time points after delivery of pEGFPUC/LF2000 packets to HeLa cells for transfection efficiency (A) or number of pDNAs per cell nucleus (B) based on Chisquare test with an $\alpha=0.05$. Data are reported as the mean $\pm \mathrm{SEM}$ $(n=6)$; see[42] for methods. Reprinted with permission from John Wiley and Sons under license number 3416000858907 [42].

proteins, which is appropriately modeled as a digital process based on control theory and systems engineering [47]. For the telecommunications model of gene delivery, queues were used to represent nonviral gene delivery where input variables arrive randomly and are processed randomly by the system (and thus output by the system randomly) [41]. Our pharmacokinetic telecommunications model using queuing theory was recently described (Fig. 1; [42]) and was able to fully recapitulate the in vitro environment for delivery of pEGFPLuc transgene packed by Lipofectamine 2000 (LF2000) nonviral carrier to HeLa cervical cancer epithelial cells in terms of transfection efficiency and unpacked nuclear pDNA (Fig. 2). Transfection efficiency refers to the portion of total cells expressing at least three transgenic green fluorescent proteins (GFPs).

In queuing networks, packets are routed through many servers, which act to perform processing tasks on the packets to ensure routing of the packet to a final destination. In such queuing networks (Fig. 3), the arrival of the packets is modeled as Poisson process, and this also works for gene delivery, e.g., 


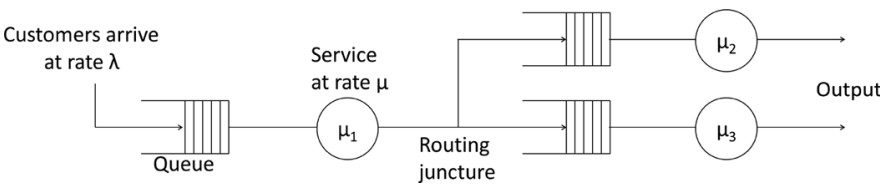

Fig. 3. Example of a two-stage queuing network with random routing [48].

in [26] where Poisson process was used to model the internalization of pDNA into a cell to be transfected, while the service time is modeled as an exponential distribution to describe the length of inter-arrival times in the Poisson process of arrivals.

In such a process, the number of arrivals in any given time interval $(\mathrm{t}, \mathrm{t}+\tau]$ follows a Poisson distribution with a parameter $(\lambda \tau)$, such that:

$$
P[(N(t+\tau)-N(t))=k]=\frac{e^{-\lambda \tau}(\lambda \tau)^{k}}{k !}
$$

where $\mathrm{N}(\mathrm{t}+\tau)-\mathrm{N}(\mathrm{t})=\mathrm{k}$ is the number of arrivals in the interval $(\mathrm{t}, \mathrm{t}+\tau)$.

The length of time it takes for the server to process the packet is described by the exponential distribution using the probability distribution of a random variable $\mathrm{X}$ in terms of the rate parameter $\lambda$ as follows:

$$
f(x ; \lambda)= \begin{cases}\lambda e^{-\lambda x} & x \geq 0 \\ 0 & x<0 .\end{cases}
$$

In general, all molecules, including pDNA, are thought to be processed by the cell in a parallel fashion, with no known limits as far as the number of molecular servers (e.g., endosomes) is concerned, to the best of the authors' knowledge. Hence this process has been modeled in our previous works [40], [42], [49] as $\mathrm{M} / \mathrm{M} / \infty$ queue. The processing rates are given in literature as the so-called kinetic constants $\mu$, being a ratio of the number of molecules processed by a single molecular server in a certain time interval to the duration of that interval 1 (Table I), usually a second, much like the rates of chemical reactions (e.). If the value of a given constant $\mu$ satisfies $0 \leq \mu \leq 1$, then it can be interpreted as a probability that a molecule can be processed by that molecular server in one second interval. If $\mu>1$, then one can consider a shorter time interval so the scaled constant $\mu^{\prime}$ satisfies $0 \leq \mu^{\prime} \leq 1$. Therefore, if there are $\mathrm{n}$ molecules to be processed at a given routing stage, the probability $p_{n}$ that at least one molecule will be processed in that scaled time interval is:

$$
p_{\mathrm{n}}=1-\left(1-\mu^{\prime}\right)^{n} .
$$

On the other hand, the probability that under the same conditions that just one molecule will be processed in that scaled time interval $\mathrm{p}_{\mathrm{n}(1)}$ is represented as:

$$
p_{\mathrm{n}(1)}=n \mu^{\prime}\left(1-\mu^{\prime}\right)^{n-1} .
$$

If the time interval is chosen small enough that $\mathrm{n} \mu^{\prime} \ll 1$, then the probability $\mathrm{p}_{\mathrm{n}(1)} \approx \mathrm{n} \mu^{\prime}$, and the scenario of an $\mathrm{M} / \mathrm{M} / \infty$ queue having a service rate of $\mu^{\prime}$ can be simplified to an $M / M / 1$ queue with a service rate of $\mu_{\mathrm{n}(1)}=\mathrm{p}_{\mathrm{n}(1)}$.

The scenario when a routing juncture, like the one shown in Fig. 1 is concerned, often happens in routing pDNA packets (see
TABLE I

Kinetic Constants Used as Service Rates in Model (See Fig. 4)

\begin{tabular}{|c|c|}
\hline Service rate & $\begin{array}{c}\text { Value } \\
{\left[\mathbf{s}^{-1}\right]}\end{array}$ \\
\hline$\mu_{1}$ & 1.45 \\
\hline$\mu_{2}$ & $1.7 \times 10^{-4}$ \\
\hline$\mu_{3}$ & $3.3 \times 10^{-4}$ \\
\hline$\mu_{4}$ & $1.7 \times 10^{-3}$ \\
\hline$\mu_{5}$ & $3.5 \times 10^{-3}$ \\
\hline$\mu_{6}$ & $5 \times 10^{-2}$ \\
\hline
\end{tabular}

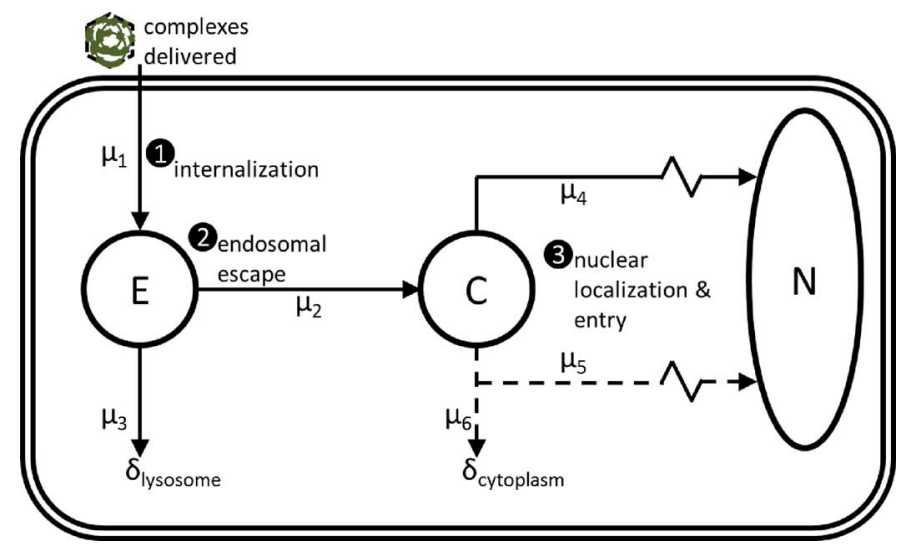

Fig. 4. Primary transmission chain between cell membrane and nucleus. Each $\mu$ parameter describes the kinetic rate $\left(\mathrm{s}^{-1}\right)$ at which a process occurs. See Fig. 2 and [42] and Table I. Solid line or dashed line indicates transmission path for pDNA packets or pDNA (unpacked state), respectively. $\delta$ indicates source of data loss during transmission of the pDNA. E: endosome; C: cytoplasm as an abstracted node; $\mathrm{N}$ : nucleus.

Fig. 1). However, a more realistic model of such a case is when a single buffer is served by two different servers - server 1 with a service rate of $0 \leq \mu_{1} \leq 1$, and a server 2 with a service rate of $0 \leq \mu_{2} \leq 1$, and the output of those 2 servers are connected to two different routes. This is a classic case of an $\mathrm{M} / \mathrm{M} / 2$ queue. In such a case, a probability $\mathrm{p}_{1,2}$ that a single pDNA packet is going to be processed by one of the servers, but not by both, equals to:

$$
\begin{aligned}
p_{1,2} & =\mu_{1}\left(1-\mu_{2}\right)+\mu_{2}\left(1-\mu_{1}\right) \\
& =\mu_{1}+\mu_{2}-2 \mu_{1} \mu_{2} .
\end{aligned}
$$

The probability $\mathrm{p}_{1}$ that the packet will be processed by the server 1 , and thus routed through the route 1 is then found using a conditional probability formula as:

$$
p_{1}=\frac{\mu_{1}-\mu_{1} \mu_{2}}{\mu_{1}+\mu_{2}-2 \mu_{1} \mu_{2}}
$$

and the probability $\mathrm{p}_{2}$ that the packet will be processed by the server 2 , and thus routed through the route 2 is given by

$$
p_{2}=\frac{\mu_{2}-\mu_{1} \mu_{2}}{\mu_{1}+\mu_{2}-2 \mu_{1} \mu_{2}} .
$$

When both $\mu_{1} \ll 1$ and $\mu_{2} \ll 1$, then the terms $\mu_{1} \mu_{2}$ and $2 \mu_{1} \mu_{2}$ can be neglected in (5)-(7), which means that it is very unlikely that in a given time slot the $\mathrm{M} / \mathrm{M} / 2$ queue could serve two pDNA packets through both outputs instantaneously. In the implemented model, we considered only such cases. It should be noted here that both $\mu_{1}$ and $\mu_{2}$ can be approximated using formula (4) if more than 1 pDNA packet is in the queue. 
For nonviral gene delivery, the kinetic constants (Table I) are reported as averages in trafficking studies in literature [15], [18], [19], [22]-[24], [44], [50]-[57]. To make the simulation model more realistic, random noise is added to the constants by using a $10 \%$ standard deviation applying a Gaussian distribution to account for the variability reported in the literature, which is presumably due to a variety of factors including differences in cell culture conditions and packet formulations. However, this value can be easily changed in the simulation environment and doing so could provide insight into variability in transfection outcomes.

Servicing of queues with a single output (Fig. 1; Fig. 2) was implemented in MATLAB using the pseudo code:

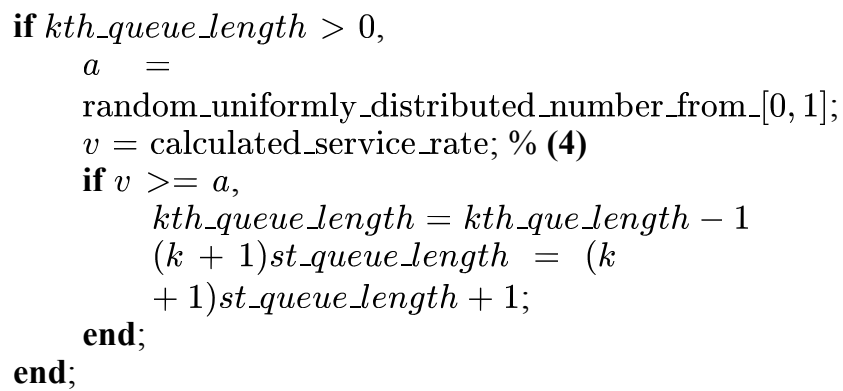

When two outputs from the server exist (Fig. 1; Fig. 2), the route was chosen randomly but with probabilities influenced by the current state of the network [58] as in (6) and (7) the rates $\mu_{1}$ and $\mu_{2}$ depend on the number of packets in the queue following (4). The queue was implemented in MATLAB with the following pseudo code for two distinct outputs serviced with the rates $v 1$ and $v 2$ :

if kth_queue_length $>0, a=$ random_uniformly_distributed_number_from_ $[0,1]$; $v 1=$ calculated_service_rate_for_output_1; \% (4)

$v 2=$ calculated_service_rate_for_output_2; \% (4)

$v r=v 1+v 2-2 * v 1 * v 2 ; \% \mathbf{~ ( 5 ) ~}$

if $v r>=a$,

$k t h \_q u e u e \_l e n g t h=k t h \_q u e \_l e n g t h-1$

$b=$

random_uniformly_distributed_number_from_ $[0,1]$; if $v 1<v 2$,

if $(v 1-v 1 * v 2) / v r<b, \%(\mathbf{6})$

$(k+1)$ st_queue_length $=(k$

else

$+1)$ st_queue_length +1 ;

$(k+2) n d_{-} q u e u e \_l e n g t h=(k$

end;

$+2)$ nd_queue_length +1 ;

else

if $(v 2-v 1 * v 2) / v r<b, \% \mathbf{~ ( 7 ) ~}$

$(k+2) n d \_q u e u e \_l e n g t h=(k$

else

$+2) n d \_q u e u e \_l e n g t h+1$;

$(k+1)$ st_queue_length $=(k$

end;

$+1)$ st_queue_length +1 ;

end;

end;

end;
In summary, our telecommunications model uses a Poisson distribution of discrete (i.e., integer) numbers of packets in a memoryless Markov process, in that the current state of the packets in the system will only affect the current iteration of the simulation. The decision for routing among multiple routes within the network is random. Finally, the model is stochastic since Gaussian noise is added to each pharmacokinetic parameter. The development and specific application of queuing theory and the telecommunications model in terms of nonviral gene delivery is also described in our earlier works [40], [42], [49].

\section{SYSTEM DESCRIPTION}

The nanocommunication system of nonviral gene delivery consists of three components: 1) pDNA: the nanosized piece of information that encodes for the reporter gene, here GFP; 2) Wrapper: the pDNA is mixed with LF2000 to electrostatically form nanosized pDNA packets; and 3) Transmission medium: the pDNA packets are then delivered to micrometer-sized HeLa human epithelial cervical cancer cells, where each cell contains molecules that act to route the pDNA (packed or unpacked; Fig. 2), thereby acting as a packet-switched network [42].

Transfection success, i.e., transfer of the pDNA into the nucleus and the subsequent production of the GFP protein encoded by the pDNA within the cell, is dictated by a series of molecular events that occur in two stages: primary transmission and secondary transmission. Primary transmission begins once pDNA packets are delivered to the cell. Upon arriving to the cell membrane, the pDNA packets are first internalized with the cell into an endosome (vesicle responsible for internalization), then escape the endosome, transit through the cytoplasm and finally localize and enter to the cell nucleus (Fig. 2; Fig. 4). pDNAs can enter the nucleus in a packed state (i.e., pDNA packets) or unpacked state (i.e., dissociated from the pDNA carrier; pDNAs). The first step of primary transmission is internalization (Fig. 4), a process that begins over the ensuing hours after pDNA packets are delivered to the media surrounding the cells in vitro. During the internalization step, nearly all pDNA packets that bind to the cell enter the cell through an endocytic pathway [25], [26]. On average, a dose of 100000 pDNA packets containing approximately 1 million pDNAs are delivered to each cell in vitro, but not all pDNA packets are internalized. Simulation results from the model [42] show within 3.9 hours the peak number of pDNAs observed in the endosomes of each cell is on average $2.8 \times 10^{4}$ (Fig. 5). The drastic drop-off of pDNAs within the endosomes after 4 hours is due to the removal of the supply of pDNA packets in vitro, accomplished by removing the media surrounding the cells and any pDNA packets not internalized, and therefore this situation is simulated in the same manner in silico.

The second step of primary transmission is endosomal escape (Fig. 2; Fig. 4), where after internalization a fraction of the pDNA packets within endosomes are able to escape into the cytoplasm. Simulation results from the model indicate the peak number of cytoplasmic pDNAs in each cell is on average $1.2 \times 10^{4}$ around 4.1 hours after delivery (Fig. 5). Those pDNA packets that do not escape from the endosomes are destroyed in lysosomes. 


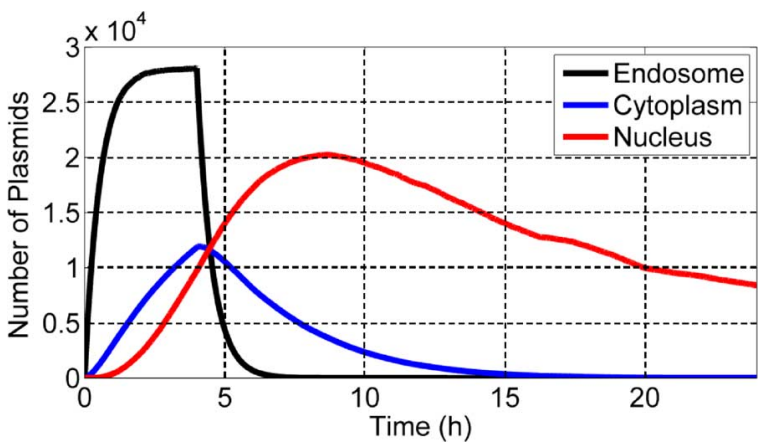

Fig. 5. During primary transmission the pDNA are distributed to the endosome, cytoplasm, and nucleus in varying quantities over time.

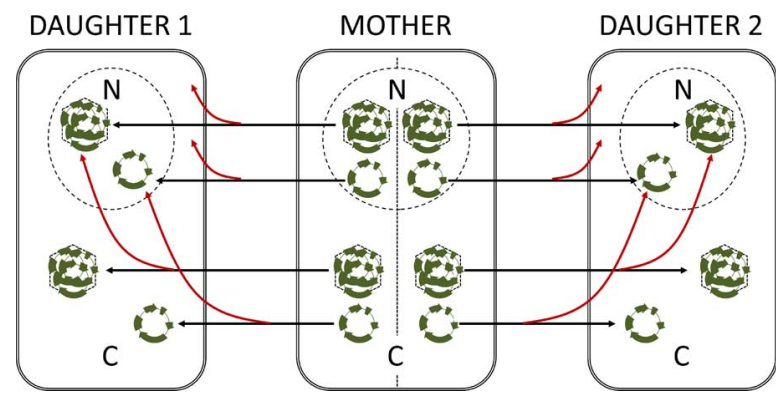

Fig. 6. Secondary transmission chain occurs during cell division when pDNA packets or pDNAs route from the nucleus of the mother cell to each daughter cell. Red arrows indicate pathways that are partially utilized (see text for explanation). Any pDNAs (green circles) or pDNA packets (green circles enclosed in dashed hexagon) in the cytoplasm route as in primary communication chain after mitosis. C: cytoplasm as an abstracted node; $\mathrm{N}$ : nucleus.

The third step of primary transmission is nuclear localization and entry (Fig. 2; Fig. 4), where pDNAs in the cytoplasm must route to and enter the cell nucleus. The pDNA can route to the nucleus in one of two states: packed or unpacked. Packed pDNA simply refers to pDNA that is still associated with the wrapper LF2000 (i.e., pDNA packet); pDNA packets are protected from degradation by nucleases in the cytoplasm. Unpacked pDNA refers to pDNA that dissociates from LF2000 (i.e., pDNA), which while required for transfection, exposes the pDNA in transit to the nucleus to degradation by nucleases within the cytoplasm. In the cytoplasm, the pDNAs, in unpacked or packed state, are then bound by nuclear localization sequence (NLS)-containing cytoplasmic proteins [15], [26], which shuttle the pDNAs into the nucleus. Simulation results from the model show a peak number of $2.0 \times 10^{4} \mathrm{pDNAs}$ arrive to the cell nucleus 9.2 hours after the cell is initially exposed to the pDNA packets (Fig. 5).

Secondary transmission occurs during cell division, when the mother cell divides and distributes the pDNA packets and pDNAs to each of two daughter cells (Fig. 6). Our telecommunications model [42] is the first to incorporate mitosis into a model of nonviral gene delivery, even though mitosis has been shown to greatly affect transfection success [21]. Since the mitosis event occurs approximately once every $18 \mathrm{~h}$, on a per cell basis, the number of cells in the experiment will increase over time. In the case when the experiment begins with 0.5 $\times 10^{4}$ cells, within $48 \mathrm{~h}$ the number of cells in the experiment has grown to $1.8 \times 10^{4}$ (Fig. 7).

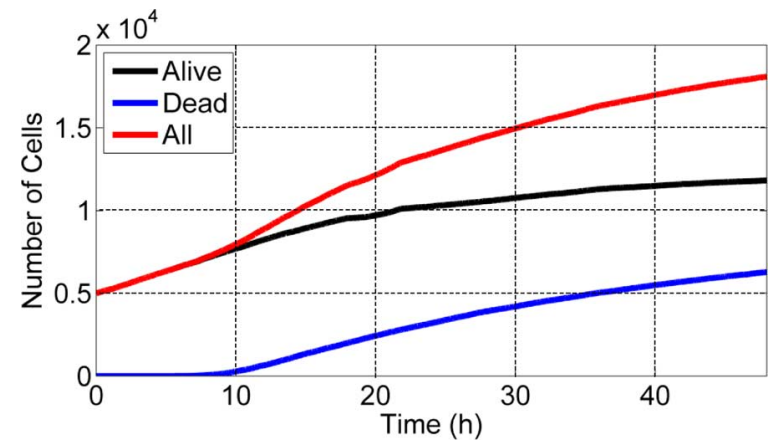

Fig. 7. Mitosis and toxicity effects on the number of cells in the experiment.

Within the secondary transmission chain, the pDNA packets and pDNAs from the mother cell is distributed to the two newly formed daughter cells (Fig. 6). After the mother cell splits, the two daughter cells start their life as non-transfected cells with no nucleus. Within 20 to 30 minutes the daughter cells have completed mitosis and formed their nuclei. Therefore, pDNAs that route from the mother cell are exposed to the cytoplasm of the daughter cells for a short period of time, and subject to the same degradation that occurs during primary transmission. However, it is not well understood how pDNAs are sorted into the nucleus or cytoplasm of the daughter cells based on if the pDNA was in the nucleus or cytoplasm of the mother cell, respectively. About $75 \%$ of those pDNAs that are in the nucleus of the mother cell will tend to also be in the nuclei of the daughter cells after a mitosis event (Fig. 6) [26]. The other $\sim 25 \%$ ends up in the cytoplasm of the daughter cells. Likewise, about $75 \%$ of pDNAs that were in the cytoplasm of the mother cell will tend to remain in the cytoplasm of daughter cells (Fig. 6) [26]. The other $\sim 25 \%$ ends up in the nucleus of the daughter cells. The distribution of the pDNA acts in the same manner whether the pDNA is packed or unpacked.

When the primary transmission chain is successful, pDNAs that are located in the cell nucleus may overproduce the encoded protein, to the point that the cell dies. Our model [42] is the first to include this biological effect, even though toxicity is observed in vitro and in vivo. Dead cells are observed in the simulations starting $\sim 10 \mathrm{~h}$ after delivery of the pDNA packets (Fig. 7). Around $0.6 \times 10^{4}$ cells die during the experiment. Those cells are removed from the experiment and no longer accounted for in calculations. The toxicity effect on cell death has been quantified in our previous work, which showed a dependency of GFP quantity on cell death. Based on experimental results and using curve fitting techniques, we previously approximated the probability that a transfected cell dies after producing $n$ molecules of GFP [42] $P_{D}(n)$ as:

$$
P_{D}(n)= \begin{cases}0, & n \leq 500 \\ \operatorname{erf}\left(\frac{n-500}{2000}\right), & n>500 .\end{cases}
$$

The plot of $P_{D}(n)$ shows that the probability of cell death increases asymptotically from $0 \%$ to $100 \%$ as the number of relative GFP molecules increases from 500 to 4000 (Fig. 8). The biological cause of the death effect is unknown; however, reports indicate cells elicit cell stress after uptake of foreign DNA [59]. Additionally, protein production is one of the highest energy demanding operations on the cell and overproduction of a 


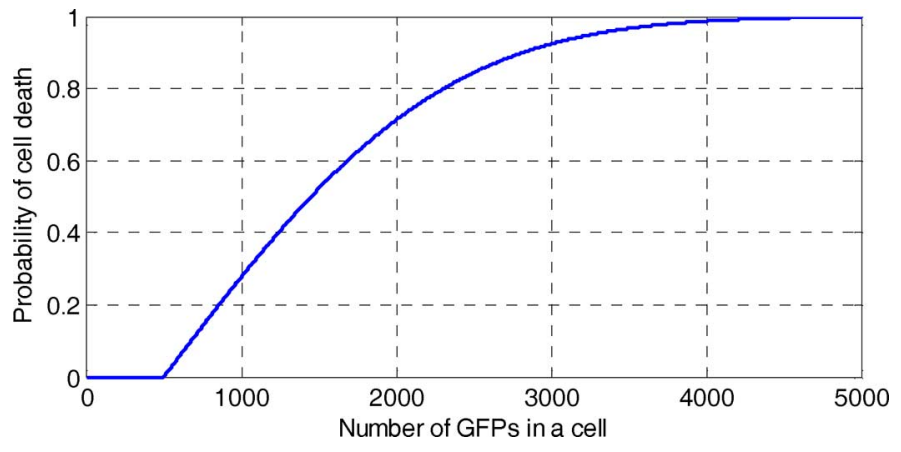

Fig. 8. Probability of treatment-induced necrosis (i.e., cell death) at a given 0.5 second time interval as a function of a number of GFPs inside that cell.

foreign protein may result in a state where the cell utilizes its resources in an unrecoverable manner, and therefore dies [60].

Taken together, we have used our nonviral gene delivery model to quantify the subcellular distribution of pDNAs during the experiment, including distribution to daughter cells during mitosis. Additionally, we quantify the increase or decrease of cells in the experiment due to mitosis or toxicity-induced cell death, respectively. Finally, we provide a mathematical relationship describing the probability of cell death based on the amount of GFP overproduction. During the gene delivery process, degradation of the pDNA can occur. That degradation is what limits the effectiveness of nonviral gene delivery systems; therefore, we next describe the primary and secondary transmission losses and offer suggestions which may lead to increased transfection.

\section{PRIMARY TRANSMISSION LOSSES}

The desired outcome when cells are exposed to the pDNA packets is for each cell to direct the pDNAs to the cell nucleus and produce the protein in relevant levels for applications in gene therapeutics [61], [62], diagnostics and functional genomics [63], [64], medical devices [65], and tissue engineering [66], [67]. However, the primary transmission as described (Fig. 3) results in lost pDNA packets due to the lysosome degradation and cytoplasmic degradation as the pDNA routes to the nucleus. To quantify only primary transmission chain losses, we removed mitosis and toxicity effects from the model to remove any pDNA degradation due to secondary transmission losses. Of the $\sim 1 \times 10^{6}$ pDNAs initially delivered to each cell, about $1.4 \times 10^{4}$ pDNAs are degraded in the lysosome by $\sim 5$ hours, indicating these pDNAs failed to escape the endosome and localize to the cytoplasm (Fig. 9). Of those pDNA packets and pDNAs which escape the endosome, another $3.4 \times 10^{4}$ pDNAs are degraded en route to the nucleus by $\sim 15$ hours after initial exposure of pDNA packets to the cell (Fig. 9). After $15 \mathrm{~h}$, the degradation plateaus because DNA not degraded in the lysosome or cytoplasm is routed to the nucleus, where the pDNA is no longer exposed to enzymatic degradation (Fig. 9).

These results suggest that limiting the degradation of the pDNAs prior to their arrival at the nucleus would lead to improved transfection, especially limiting lysosomal degradation, which our previous work [42] identified to be the most sensitive parameter affecting transfection success. Therefore,

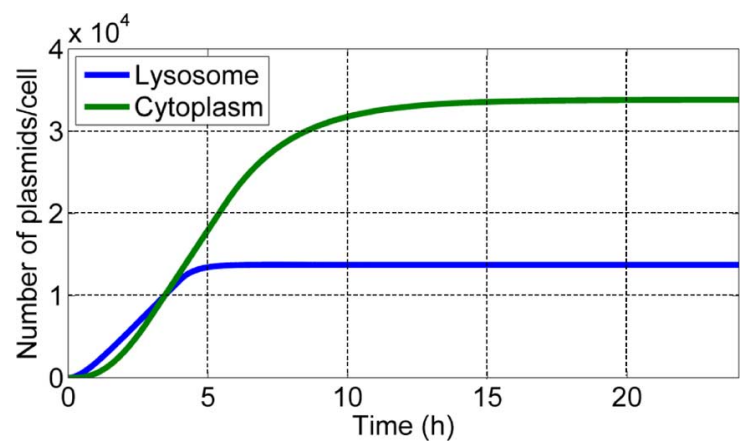

Fig. 9. pDNAs are lost in the lysosome and cytoplasm during routing to the cell nucleus.

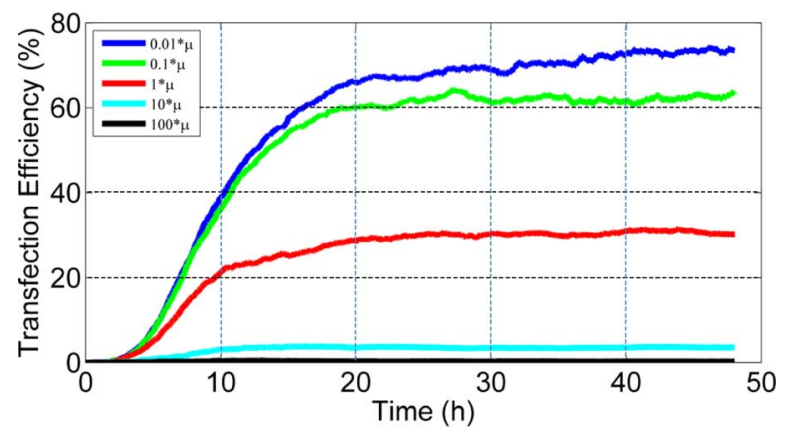

Fig. 10. Increasing $(0.01 * \mu, 0.1 * \mu)$ or decreasing $(10 * \mu, 100 * \mu)$ endosomal escape kinetics results in increased or decreased transfection efficiency, respectively, compared to baseline $(1 * \mu)$.

in this work, we explored the ramifications of improving the gene delivery system to limit pDNA losses at the lysosomal degradation stage in data transmission. Specifically, we evaluated the effect on transfection efficiency, which is the number of cells expressing GFP divided by the total number of cells in the experiment. In our simulations, enhancing the endosomal escape kinetics by 10 -fold or 100-fold faster (and thus limiting the transfer of pDNA from the endosome to the lysosome) resulted in increases in transfection efficiency from $\sim 30 \%$ to $60 \%$ or $70 \%$, respectively, $48 \mathrm{~h}$ after delivery of pDNA packets (Fig. 10).

For the 100-fold enhancement to endosomal escape, two-fold more pDNAs were observed in the nucleus of the cell. Physicochemical modifications to the DNA carrier which would achieve increased endosomal escape kinetics and improved transfection may be dodecylation [22] or incorporation of a $\mathrm{pH}$-sensitive fusogenic peptide [68], two modifications previously shown to improve transfection.

The amount of pDNAs successfully delivered to the nucleus plays a crucial role in transfection levels. Those cells which contain a higher number of nuclear pDNAs exhibit increased transfection, though with a saturating effect around $1 \times 10^{4}$ nuclear pDNAs [69]. Unfortunately, as aforementioned, most pDNAs are degraded before trafficking to the nucleus, thereby suffering large transmission losses. Therefore, we next explored model simulations showing the effect of the number of nuclear pDNAs on probability of transfection. From [69] we have developed a heuristic estimate for the probability that at a given time interval, a particular pDNA transfects the cell. Assuming a 0.5 second 

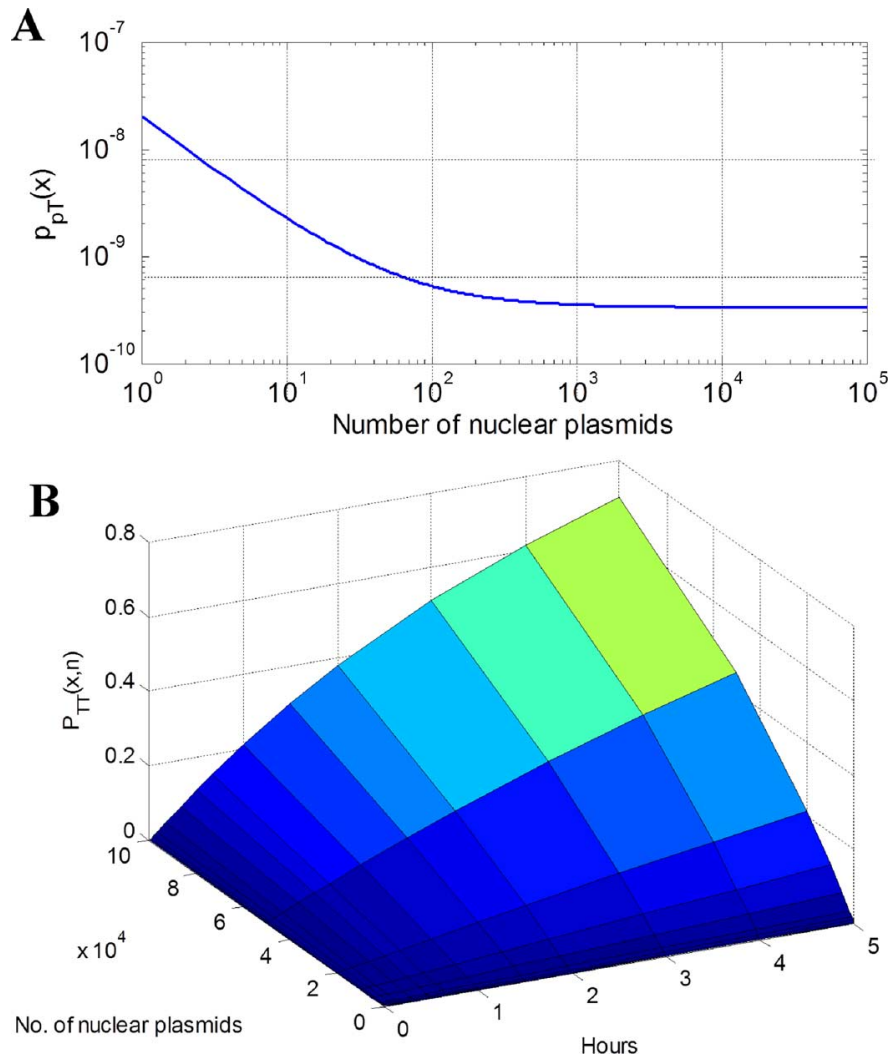

Fig. 11. Probabilities of successful data transmission (i.e., transfection). (a) Probability of transfection by a particular pDNA. (b) Probability of transfection over time by varying amount of pDNAs in nucleus.

time interval and $k=x$ pDNAs present in nucleus, that probability is given by

$$
P_{p T}\{k=x\}=\frac{1.95 \cdot 10^{-8}}{x}+3.33 \cdot 10^{-10} .
$$

Assuming that events of transfecting a cell by different pDNA are statistically independent, the total probability that a cell becomes transfected at a particular 0.5 second time interval in the presence of $k=x$ pDNAs in the nucleus is then found using the Bernoulli formula as

$$
P_{T}\{k=x\}=1-\left[1-P_{p T}\{k=x\}\right]^{x}
$$

and the probability that the cell becomes transfected after $n$ successive time intervals equals to:

$$
P_{T T}\{k=x, n\}=1-\left\{1-\left[1-P_{T}\{k=x\}\right]^{x}\right\}^{n} .
$$

The plots of $P_{p T}$ [70] and $P_{T T}$ [70] are shown in Fig. 11(a) and (b), respectively. The efficiency of transfection by a particular pDNA drops as the number of pDNA increases, with a saturating effect of $\mathrm{p}=8 \times 10^{-10}$ or around $10^{2} \mathrm{pDNAs}$ (Fig. 11(a)). The biological significance of the saturation could be due to availability of cell resources to transcribe the pDNA and convert its code into the encoded protein. Additionally, those cells with a high number of pDNAs also contain high quantities of the DNA carrier, which has been shown to impede mRNA activity (loss in ability to transcode the information) [71] and nuclear import (loss in transmission signal) [72], thereby acting to reduce efficiency of transfection on a per pDNA basis.
The amount of pDNA present in the nucleus also affects transfection levels in a time-dependent manner. After $5 \mathrm{~h}$, the probability of transfection increases almost linearly from 0 to $70 \%$ as the number of pDNAs increases from 0 to $10 \times 10^{4}$ (Fig. 11(b)). Likewise at $10 \times 10^{4}$ pDNAs per nucleus, the probability of transfection increases almost linearly from 0 to $70 \%$ as the duration the pDNAs are in the nucleus increases from $0 \mathrm{~h}$ to $5 \mathrm{~h}$. The result highlights the importance of having high numbers of pDNAs in the nucleus, also shown by Ludtke and colleagues [73], where increasing the number of nuclear pDNAs above 571 per cell was enough to achieve $\sim 100 \%$ transfection. One way to increase the amount of nuclear pDNA is to reduce pDNA losses to the lysosome (Fig. 10; and data not shown). However, increased pDNAs in the nucleus lead to increased GFP production (data not shown), and as a consequence, increased probability of treatment induced necrosis (Fig. 8).

Taken together, we have characterized primary transmission pDNA losses in the lysosome (due to failure of endosomal escape) and in the cytoplasm (for those pDNAs en route to the nucleus) and have shown that increasing pDNA quantities in each compartment or time in the nucleus results in increasing probabilities of transfection. We also demonstrated that reducing pDNA losses due to lysosomal degradation results in increased transfection, though at risk of increased probability of cell death due to GFP accumulation.

\section{Secondary Transmission Losses}

While primary pDNA transmission loses in the lysosome and cytoplasm are more well-known in the literature, secondary transmission losses may occur during a mitosis event, when the mother cell divides into two daughter cells. During mitosis, contents of the mother cell are distributed to two daughter cells (including pDNA packets, pDNAs and transgenic mRNAs, and transgenic GFPs; Fig. 2) and a new nuclear membrane is rebuilt in each daughter cell. Because there is no nucleus during mitosis, the unpacked pDNAs may be exposed to degradation in the cytoplasm. To account for pDNA degradation during mitosis, the same kinetic constant was applied as for pDNA degradation during the trafficking of unpacked pDNAs to the nucleus through the cytoplasm during primary transmission. Such pDNA losses after mitosis, used in our model, are consistent with those reported in in vitro transfection [26]. Therefore, secondary transmission pDNA losses occur during the mitosis period as a result of distribution of pDNAs from mother cell to the two daughter cells. Between $5 \mathrm{~h}$ and $24 \mathrm{~h}$, approximately $30 \%$ of pDNAs are lost by the end of the mitosis, leaving on average 10000 pDNAs in the cell (Fig. 12). In addition each mitosis event acts to dilute the number of pDNAs per cell, because the number of cells in the experiment increases and affects the calculation of transfection efficiency. Meanwhile, the accrued number of degraded pDNAs remains constant per cell due to exposure to cytoplasmic nucleases during each mitosis event. Therefore, by $48 \mathrm{~h}$, the number of surviving pDNAs is around 4000 per cell (Fig. 12). The dilution of nuclear pDNAs and loss of nuclear pDNAs during mitosis is consistent with in vitro observations [26].

The pDNAs lost during this time period highlights an unexplored area where nonviral gene delivery systems could be im- 


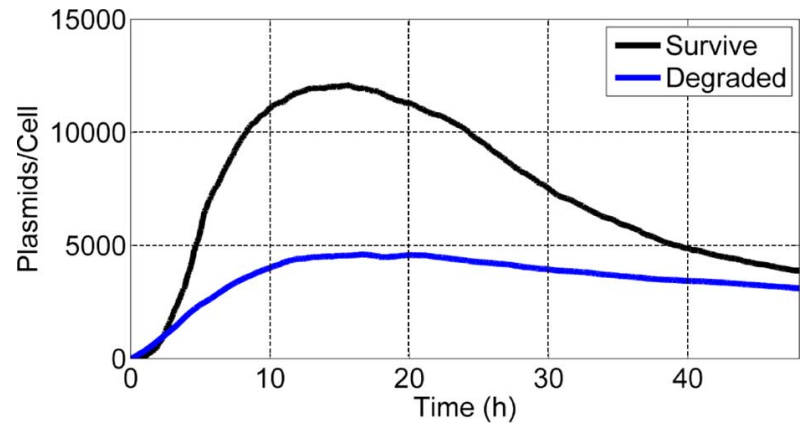

Fig. 12. As a cell undergoes mitosis, the pDNAs are exposed to degradation in the cytoplasm during distribution to daughter cells resulting in a portion of pDNAs that are degraded during secondary transmission.

proved. For example, the pDNA could be protected from degradation by targeting antibodies to discrete portions of the pDNA, thereby providing steric hindrance of DNase degradation [74].

\section{CONCLUSIONS}

Nonviral gene delivery systems, which are designed to deliver pDNA encoding proteins to cells, must be improved in order to achieve a relevant therapeutic effect. Losses in the transmission of pDNAs prevent therapeutic protein production. In this work, we quantify those pDNA losses that occur during primary transmission of the pDNA to the nucleus and in secondary transmission of pDNA from mother cell to daughter cells. Limiting those pDNA losses can increase the number of pDNAs which make it to the nucleus, and therefore increase transfection in a dose- and time-dependent manner. However, increased number of nuclear pDNAs leads to increased transgene production and, therefore, an increased probability of cell death due to toxicity. One mechanism to prevent the toxicity could be to reduce the frequency of transgene production (such as by using a weaker promoter). An increase in pDNAs in the nucleus would also act to reduce the diluting effect of pDNA caused by mitosis and provide sustained gene expression. Therefore, the design of new gene delivery systems should consider the trade-off in quantity of nuclear pDNA, mitosis, and toxicity in order to achieve therapeutically relevant transfection levels.

\section{ACKNOWLEDGMENT}

The authors have no conflicts of interest to declare.

\section{REFERENCES}

[1] T. Nakano, M. J. Moore, F. Wei, A. V. Vasilakos, and J. W. Shuai, "Molecular communication and networking: Opportunities and challenges," IEEE Trans. NanoBiosci., vol. 11, pp. 135-148, Jun. 2012.

[2] I. F. Akyildiz, F. Brunetti, and C. Blazquez, "Nanonetworks: A new communication paradigm," Comput. Netw., vol. 52, pp. 2260-2279, Aug. 2008.

[3] S.-I. Nakano and M. S. Rahman, "WALCOM : Algorithms and computation," in Proc. 2nd Int. Workshop (WALCOM 2008), Dhaka, Bangladesh, Feb. 7-8, 2008.

[4] P1906.1-Recommended Practice for Nanoscale and Molecular Communication Framework, , 2013 [Online]. Available: http://standards. ieee.org/develop/project/1906.1.html
[5] Y. Moritani, S. x. S. Hiyama, and T. Suda, "Molecular communication for health care applications," in Proc. 4th Annu. IEEE Int. Conf. Pervasive Comput. Commun. Workshops, 2006.

[6] F. Walsh et al., "Hybrid DNA and enzyme based computing for address encoding, link switching and error correction in molecular communication," in Nano-Net., M. Cheng, Ed. Berlin/Heidelberg, Germany: Springer, 2009, vol. 3, pp. 28-38.

[7] M. Pierobon and I. F. Akyildiz, "A physical end-to-end model for molecular communication in nanonetworks," IEEE J. Sel. Areas Commun., vol. 28, pp. 602-611, 2010.

[8] Y. H. Li et al., "A quorum-sensing signaling system essential for genetic competence in Streptococcus mutans is involved in biofilm formation," J. Bacteriol., vol. 184, pp. 2699-2708, May 2002.

[9] L. Naldini, "Ex vivo gene transfer and correction for cell-based therapies," Nat. Rev. Genet., vol. 12, pp. 301-315, May 2011.

[10] R. Heilbronn and S. Weger, "Viral vectors for gene transfer: Current status of gene therapeutics," Handb. Exp. Pharmacol., pp. 143-170, 2010.

[11] C. E. Thomas, A. Ehrhardt, and M. A. Kay, "Progress and problems with the use of viral vectors for gene therapy," Nat. Rev. Genet., vol. 4, pp. 346-358, May 2003.

[12] N. M. Rao and V. Gopal, "Cell biological and biophysical aspects of lipid-mediated gene delivery," Biosci. Rep., vol. 26, pp. 301-324, Aug. 2006.

[13] B. P. Chen, C. D. Wolfgang, and T. Hai, "Analysis of ATF3, a transcription factor induced by physiological stresses and modulated by gadd153/Chop10," Mol. Cell. Biol., vol. 16, pp. 1157-1168, Mar. 1996.

[14] S. K. Lai, K. Hida, C. Chen, and J. Hanes, "Characterization of the intracellular dynamics of a non-degradative pathway accessed by polymer nanoparticles," J. Control Release, vol. 125, pp. 107-111, Jan. 2008

[15] C. M. Varga, K. Hong, and D. A. Lauffenburger, "Quantitative analysis of synthetic gene delivery vector design properties," Mol. Ther., vol. 4, pp. 438-446, Nov. 2001.

[16] J. Suh, D. Wirtz, and J. Hanes, "Efficient active transport of gene nanocarriers to the cell nucleus," Proc. Natl. Acad. Sci. USA, vol. 100, pp. 3878-3882, Apr. 2003.

[17] U. Jandt, S. Shao, M. Wirth, and A. P. Zeng, "Spatiotemporal modeling and analysis of transient gene delivery," Biotechnol. Bioeng., vol. 108 , pp. 2205-2217, Sep. 2011.

[18] D. A. Dean, B. S. Dean, S. Muller, and L. C. Smith, "Sequence requirements for plasmid nuclear import," Exp. Cell. Res., vol. 253, pp. 713-722, Dec. 1999.

[19] G. Schwake et al., "Predictive modeling of non-viral gene transfer," Biotechnol. Bioeng., vol. 105, pp. 805-813, Mar. 2010.

[20] W. T. Godbey, K. K. Wu, and A. G. Mikos, "Poly(ethylenimine) and its role in gene delivery," J. Control Release, vol. 60, pp. 149-160, Aug. 1999.

[21] S. Brunner, T. Sauer, S. Carotta, M. Cotten, M. Saltik, and E. Wagner, "Cell cycle dependence of gene transfer by lipoplex, polyplex and recombinant adenovirus," Gene Ther., vol. 7, pp. 401-407, Mar. 2000.

[22] C. M. Varga, N. C. Tedford, M. Thomas, A. M. Klibanov, L. G. Griffith, and D. A. Lauffenburger, "Quantitative comparison of polyethylenimine formulations and adenoviral vectors in terms of intracellular gene delivery processes," Gene Ther., vol. 12, pp. 1023-1032, Jul. 2005.

[23] O. Zelphati and F. C. Szoka Jr., "Mechanism of oligonucleotide release from cationic liposomes," Proc. Natl. Acad. Sci. USA, vol. 93, pp. 11493-11498, Oct. 1996.

[24] K. Lappalainen, R. Miettinen, J. Kellokoski, I. Jaaskelainen, and S. Syrjanen, "Intracellular distribution of oligonucleotides delivered by cationic liposomes: Light and electron microscopic study," $J$. Histochem Cytochem, vol. 45, pp. 265-274, Feb. 1997.

[25] A. Elouahabi and J. M. Ruysschaert, "Formation and intracellular trafficking of lipoplexes and polyplexes," Mol. Ther., vol. 11, pp. 336-347, Mar. 2005.

[26] J. Z. Gasiorowski and D. A. Dean, "Postmitotic nuclear retention of episomal plasmids is altered by DNA labeling and detection methods," Mol. Ther., vol. 12, pp. 460-467, Sep. 2005.

[27] T. Blessing, M. Kursa, R. Holzhauser, R. Kircheis, and E. Wagner, "Different strategies for formation of pegylated EGF-conjugated PEI/DNA complexes for targeted gene delivery," Bioconjug. Chem., vol. 12, pp. 529-537, Jul.-Aug. 2001.

[28] M. Ogris, P. Steinlein, S. Carotta, S. Brunner, and E. Wagner, "DNA/ polyethylenimine transfection particles: Influence of ligands, polymer size, and PEGylation on internalization and gene expression," AAPS PharmSci, vol. 3, p. E21, 2001. 
[29] E. Liang, M. N. Rosenblatt, P. S. Ajmani, and J. A. Hughes, "Biodegradable $\mathrm{pH}$-sensitive surfactants (BPS) in liposome-mediated nucleic acid cellular uptake and distribution," Eur. J. Pharm. Sci., vol. 11, pp. 199-205, Sep. 2000.

[30] J. G. Duguid et al., "A physicochemical approach for predicting the effectiveness of peptide-based gene delivery systems for use in plasmidbased gene therapy,” Biophys. J., vol. 74, pp. 2802-2814, Jun. 1998.

[31] B. Atakan and O. Akan, "On channel capacity and error compensation in molecular communication," in Transactions on Computational Systems Biology X, C. Priami, F. Dressler, O. Akan, and A. Ngom, Eds. Berlin/Heidelberg, Germany: Springer, 2008, vol. 5410, pp. 59-80.

[32] F. Walsh and S. Balasubramaniam, "Reliability and delay analysis of multihop virus-based nanonetworks," IEEE Trans. Nanotechnol., vol. 12, pp. 674-684, Sep. 2013.

[33] R. W. Horobin and V. Weissig, "A QSAR-modeling perspective on cationic transfection lipids. 1. Predicting efficiency and understanding mechanisms," J. Gene Med., vol. 7, pp. 1023-1034, Aug. 2005.

[34] G. A. Banks, R. J. Roselli, R. Chen, and T. D. Giorgio, "A model for the analysis of nonviral gene therapy," Gene Ther., vol. 10, pp. 1766-1775, Sep. 2003.

[35] C. M. Roth and S. Sundaram, "Engineering synthetic vectors for improved DNA delivery: Insights from intracellular pathways," Annu. Rev. Biomed. Eng., vol. 6, pp. 397-426, 2004.

[36] R. Moriguchi, K. Kogure, and H. Harashima, "Non-linear pharmacodynamics in the transfection efficiency of a non-viral gene delivery system," Int. J. Pharm., vol. 363, pp. 192-198, Nov. 2008.

[37] A. T. Dinh, C. Pangarkar, T. Theofanous, and S. Mitragotri, "Understanding intracellular transport processes pertinent to synthetic gene delivery via stochastic simulations and sensitivity analyses," Biophys. J., vol. 92, pp. 831-846, Feb. 2007.

[38] P. Berraondo, G. Gonzalez-Aseguinolaza, and I. F. Troconiz, "Semimechanistic pharmacodynamic modelling of gene expression and silencing processes," Eur. J. Pharm. Sci., vol. 37, pp. 418-426, Jun. 2009.

[39] C. H. Jones, C. K. Chen, A. Ravikrishnan, S. Rane, and B. A. Pfeifer, "Overcoming nonviral gene delivery barriers: Perspective and future," Mol. Pharm., Oct. 2013.

[40] B. J. Wysocki, T. M. Martin, T. A. Wysocki, and A. K. Pannier, "Modeling nonviral gene delivery as a macro-to-nano communication system," Nano Commun. Netw., vol. 4, pp. 14-22, 2013.

[41] D. G. Kendall, "Stochastic processes occurring in the theory of queues and their analysis by the method of the imbedded Markov chain," $A n$ nals Math. Stat., vol. 24, pp. 338-354, 1953.

[42] T. M. Martin, B. J. Wysocki, J. P. Beyersdorf, T. A. Wysocki, and A. K. Pannier, "Integrating mitosis, toxicity, and transgene expression in a telecommunications packet-switched network model of lipoplexmediated gene delivery," Biotechnol. Bioeng., vol. 111, pp. 1659-1671, Aug. 2014.

[43] H. Akita, R. Ito, I. A. Khalil, S. Futaki, and H. Harashima, "Quantitative three-dimensional analysis of the intracellular trafficking of plasmid DNA transfected by a nonviral gene delivery system using confocal laser scanning microscopy," Mol. Ther., vol. 9, pp. 443-451, Mar. 2004.

[44] K. Hakamada and J. Miyake, "Evaluation method for gene transfection by using the period of onset of gene expression and cell division," $J$. Biosci. Bioeng., vol. 113, pp. 124-127, Jan. 2012.

[45] M. F. Neuts and S. Z. Chen, The Infinite Server Queue with Poisson Arrivals and Semi-Markovian Services. Lafayette, IN, USA: Purdue University Department of Statistics: Defense Technical Information Center, 1970, Mimeograph Series.

[46] A. T. Sharp, A. K. Pannier, B. J. Wysocki, and T. A. Wysocki, "A novel telecommunications-based approach to HIV modeling and simulation," Nano Commun. Netw., vol. 3, pp. 129-137, 2012.

[47] R. E. Ziemer, W. H. Tranter, and D. R. Fannin, Signals and Systems : Continuous and Discrete, 4th ed. Upper Saddle River, NJ, USA: Prentice-Hall, 1998.

[48] I. J. B. F. Adan, O. J. Boxma, and J. A. C. Resing, "Queueing models with multiple waiting lines," Queueing Syst., vol. 37, pp. 65-98, 2001.

[49] B. J. Wysocki, T. M. Martin, T. A. Wysocki, and A. K. Pannier, "Simulation supported estimation of end-to-end transmission parameters in non-viral gene delivery," in IEEE ICC 2014-Sel. Areas Commun. Symp., 2014, pp. 4179-4183.

[50] Z. U. Rehman, D. Hoekstra, and I. S. Zuhorn, "Mechanism of polyplexand lipoplex-mediated delivery of nucleic acids: Real-time visualization of transient membrane destabilization without endosomal lysis," ACS Nano, vol. 7, pp. 3767-3777, May 2013.
[51] D. Lechardeur et al., "Metabolic instability of plasmid DNA in the cytosol: A potential barrier to gene transfer," Gene Ther., vol. 6, pp. 482-497, Apr. 1999.

[52] G. L. Wilson, B. S. Dean, G. Wang, and D. A. Dean, "Nuclear import of plasmid DNA in digitonin-permeabilized cells requires both cytoplasmic factors and specific DNA sequences," J. Biol. Chem., vol. 274, pp. 22025-22032, Jul. 1999.

[53] J. Moroianu, G. Blobel, and A. Radu, "Nuclear protein import: Ran-GTP dissociates the karyopherin alphabeta heterodimer by displacing alpha from an overlapping binding site on beta," Proc. Natl. Acad. Sci. USA, vol. 93, pp. 7059-7062, Jul. 1996.

[54] D. A. Hume, "Probability in transcriptional regulation and its implications for leukocyte differentiation and inducible gene expression," Blood, vol. 96, pp. 2323-2328, Oct. 2000.

[55] A. Sacchetti, T. El Sewedy, A. F. Nasr, and S. Alberti, "Efficient GFP mutations profoundly affect mRNA transcription and translation rates," FEBS Lett., vol. 492, pp. 151-155, Mar. 2001.

[56] J. A. Sniegowski, J. W. Lappe, H. N. Patel, H. A. Huffman, and R. M. Wachter, "Base catalysis of chromophore formation in Arg96 and Glu222 variants of green fluorescent protein," J. Biol. Chem., vol. 280, pp. 26248-26255, Jul. 2005.

[57] B. Alberts, Molecular Biology of the Cell, 3rd ed. New York: Garland, 1994.

[58] W. Stallings, Data and Computer Communications, 8th ed. Upper Saddle River, NJ, USA: Pearson/Prentice Hall, 2007.

[59] T. M. Martin, S. A. Plautz, and A. K. Pannier, "Network analysis of endogenous gene expression profiles after polyethyleneimine-mediated DNA delivery," J. Gene Med., vol. 15, pp. 142-154, Mar.-Apr. 2013.

[60] M. C. Jewett, M. L. Miller, Y. Chen, and J. R. Swartz, "Continued protein synthesis at low [ATP] and [GTP] enables cell adaptation during energy limitation," J. Bacteriol., vol. 191, pp. 1083-1091, Feb. 2009.

[61] N. F. Sun, Z. A. Liu, W. B. Huang, A. L. Tian, and S. Y. Hu, "The research of nanoparticles as gene vector for tumor gene therapy," Crit. Rev. Oncol. Hematol., Oct. 2013.

[62] T. Niidome and L. Huang, "Gene therapy progress and prospects: Nonviral vectors," Gene Ther., vol. 9, pp. 1647-1652, Dec. 2002.

[63] A. K. Pannier, E. A. Ariazi, A. D. Bellis, Z. Bengali, V. C. Jordan, and L. D. Shea, "Bioluminescence imaging for assessment and normalization in transfected cell arrays," Biotechnol. Bioeng., vol. 98, pp. 486-497, Oct. 2007.

[64] R. Xing, G. Liu, J. Zhu, Y. Hou, and X. Chen, "Functional magnetic nanoparticles for non-viral gene delivery and MR imaging," Pharm. Res., Sep. 2013.

[65] M. Zilberman, A. Kraitzer, O. Grinberg, and J. J. Elsner, "Drug-eluting medical implants," Handb. Exp. Pharmacol., pp. 299-341, 2010.

[66] H. Lu, L. Lv, Y. Dai, G. Wu, H. Zhao, and F. Zhang, "Porous chitosan scaffolds with embedded hyaluronic acid/chitosan/plasmid-DNA nanoparticles encoding TGF-beta1 induce DNA controlled release, transfected chondrocytes, and promoted cell proliferation," PLoS One, vol. 8, p. e69950, 2013.

[67] J. L. Santos, D. Pandita, J. Rodrigues, A. P. Pego, P. L. Granja, and H. Tomas, "Non-viral gene delivery to mesenchymal stem cells: Methods, strategies and application in bone tissue engineering and regeneration," Curr. Gene Ther., vol. 11, pp. 46-57, Feb. 2011.

[68] H. Hatakeyama et al., "A pH-sensitive fusogenic peptide facilitates endosomal escape and greatly enhances the gene silencing of siRNA-containing nanoparticles in vitro and in vivo," J. Control Release, vol. 139, pp. 127-132, Oct. 2009.

[69] R. Tachibana, H. Harashima, Y. Shinohara, and H. Kiwada, "Quantitative studies on the nuclear transport of plasmid DNA and gene expression employing nonviral vectors," Adv. Drug Deliv. Rev., vol. 52, pp. 219-226, Nov. 2001.

[70] M. Bouwens et al., "Fish-oil supplementation induces antiinflammatory gene expression profiles in human blood mononuclear cells," Amer. J. Clin. Nutr., vol. 90, pp. 415-424, Aug. 2009.

[71] S. Hama, H. Akita, S. Iida, H. Mizuguchi, and H. Harashima, "Quantitative and mechanism-based investigation of post-nuclear delivery events between adenovirus and lipoplex," Nucleic Acids Res., vol. 35, pp. 1533-1543, 2007.

[72] I. Honore, S. Grosse, N. Frison, F. Favatier, M. Monsigny, and I. Fajac, "Transcription of plasmid DNA: Influence of plasmid DNA/polyethylenimine complex formation," J. Control Release, vol. 107 , pp. 537-546, Oct. 2005.

[73] J. J. Ludtke, M. G. Sebestyen, and J. A. Wolff, "The effect of cell division on the cellular dynamics of microinjected DNA and dextran," Mol. Ther., vol. 5, pp. 579-588, May 2002. 
[74] W. Emlen, R. Ansari, and G. Burdick, "DNA-anti-DNA immune complexes. Antibody protection of a discrete DNA fragment from DNase digestion in vitro," J. Clin. Invest., vol. 74, pp. 185-190, Jul. 1984.

Timothy Michael Martin received the B.S. degree in mechanical engineering and the B.S. degree in biomedical engineering in 2006, both from Rose-Hulman Institute of Technology, Terre Haute, IN, USA. He then received the Engineer in Training certificate in 2006. In 2014, he received a Ph.D. degree in biomedical engineering from University of Nebraska-Lincoln (UNL), Lincoln, NE, USA.

During and after his undergraduate studies, he served in the US Marine Corps during combat operations for Operation Iraqi Freedom and Operation Enduring Freedom in $\mathrm{Al} \mathrm{Taqaddum,} \mathrm{Iraq.} \mathrm{Also} \mathrm{after} \mathrm{his} \mathrm{undergraduate} \mathrm{studies,} \mathrm{he} \mathrm{worked}$ for four years as a process engineer at Cook Pharmica, Bloomington, IN, USA During his Ph.D. dissertation work, he received the Larrick Memorial Trave Award, NCMN Graduate Fellowship, and Outstanding Graduate Research Assistant. His research interests include gene and drug delivery for cancer therapeutics and is currently a postdoctoral fellow at University of Nebraska Medical Center, Lincoln, NE, USA, in the Department of Pharmaceutical Sciences. He is author of 4 research publications. Dr. Martin is a current member of American Institute of Chemical Engineers, American Heart Association, and Biomedical Engineering Society.

Beata Joanna Wysocki graduated from Warsaw University of Technology, Warsaw, Poland, receiving the M.Eng. degree in electrical engineering in 1991 In 1994, she started the Ph.D. degree in the Australian Telecommunications Research Institute at Curtin University of Technology. In March 2000, she was awarded her Ph.D. for the thesis "Signal Formats for Code Division Multiple Access Wireless Networks." Since October 1999 she has been with the Telecommunications \& Information Technology Research Institute at the University Wollongong as a research fellow. Her research interests include: space-time signal processing, sequence design for direct sequence (DS) code division multiple access (CDMA) data networks and optimization of ultra wideband (UWB) communication systems as well as modeling biological processes at nanoscale.

Tadeusz Antoni Wysocki (M'94-SM'98), received the M.Sc.Eng. degree with the highest distinction in telecommunications from the Academy of Technology and Agriculture, Bydgoszcz, Poland, in 1981. In 1984, he received the Ph.D. degree, and in 1990, was awarded the D.Sc. degree (habilitation) in telecommunications from the Warsaw University of Technology, Warsaw, Poland.

In 1992, he moved to Perth, Western Australia, to work at Edith Cowan University. He spent the whole 1993 at the University of Hagen, Germany, within the framework of Alexander von Humboldt Research Fellowship. In December 1998, he moved to the University of Wollongong, NSW, Australia, as an Associate Professor, within the School of Electrical, Computer and Telecommunications Engineering. Since the fall of 2007, he has been with the University of Nebraska-Lincoln, Lincoln, NE, USA, as Professor of Computer and Electronics Engineering at the Peter Kiewit Institute, Omaha, NE, USA. The main areas of his research interests include: space-time signal processing, diversity combining, indoor propagation of microwaves, and biological communications at nanoscale. He is an author or coauthor of over 250 research papers. Dr. Wysocki was an interim Chair of the newly established Chapter of IEEE Communications Society in Western Australia in 1997 and was elected to chair of that Chapter in 1998. In 2009, Dr. Wysocki was a Chair of the Nebraska Chapter of IEEE Communications Society and in 2010 and 2011 a Chair of Nebraska Chapter of IEEE Computers Society.

Angela K. Pannier received the B.S. degree and the M.S. degree in biological systems engineering from the University of Nebraska-Lincoln (UNL), Lincoln, NE, USA (in 2001 and 2002, respectively), and the Ph.D. degree in Biological Sciences from Northwestern University in Evanston, IL, USA, in 2007.

Hired in 2007 by University of Nebraska-Lincoln as an Assistant Professor, she is currently an Associate Professor in the Department of Biological Systems Engineering at UNL (since July 2013) and a William E. Brooks Engineering Leadership Fellow. She has also been appointed a member of the $\mathrm{Ne}$ braska Center for Materials and Nanoscience, the Center for Nanohybrid Functional Materials at UNL and a member of the Center for Drug Delivery and Nanomedicine at the University of Nebraska Medical Center (UNMC), Omaha, NE, USA. Dr. Pannier also holds a courtesy appointment in the Mary and Dick Holland Regenerative Medicine Program at UNMC. The author of over 25 peerreviewed articles, and two book chapters, her research interests include modeling nonviral gene delivery, developing novel nonviral gene delivery systems, and biomaterials for embryological and tissue engineering applications. Dr. Pannier is a member of the American Institute of Chemical Engineers, Biomedical Engineering Society, Society of Women Engineers, Institute of Biological Engineers, and the American Society of Gene \& Cell Therapy. 